\title{
Depression and anxiety symptoms in cardiac patients: a cross-sectional hospital- based study in a Palestinian population
}

\author{
H. Allabadi 1,2,3, A. Alkaiyat 1,2,3 , A. Alkhayyat ${ }^{3}$, A. Hammoudi ${ }^{3}$, H. Odeh ${ }^{3}$, J. Shtayeh ${ }^{3}$, M. Taha ${ }^{3}$, C. Schindler ${ }^{1,2}$, \\ E. Zemp ${ }^{1,2}$, S. Haj-Yahia ${ }^{4,5,6+}$ and N. Probst-Hensch ${ }^{1,2^{*}+}$
}

\begin{abstract}
Background: Mental health problems have an adverse effect on the course of cardiac disease. The integration of their diagnosis and treatment into cardiology care is generally poor. It is particularly challenging in cultural environments where mental health problems are stigmatized. The objective of the current study was to investigate the proportion of cardiac patients with depression and anxiety as well as factors associated with the presence of these symptoms in a Palestinian population.

Methods: This cross-sectional hospital-based study was conducted on patients consecutively admitted with a new or existing cardiac diagnosis to one of the four main hospitals in Nablus, Palestine over an eight-month period. Data was obtained from hospital medical charts and an in-person interview, using a structured questionnaire with a sequence of validated instruments. All subjects were screened for depression and anxiety using the Cardiac Depression Scale (CDS) and the Depression Anxiety Stress Scale (DASS-42). Multivariate ordered logistic regression analyses were performed to identify factors among four categories (socio-demographic, clinical, psychosocial, lifestyle) independently associated with depression and anxiety.
\end{abstract}

Results: In total, 1053 patients with a confirmed cardiac diagnosis were included in the study with a participation rate of 96\%. Based on the CDS and DASS-42, 54\% met the criteria for severe depression (CDS > 100) and 19.2\% for severe-tovery severe anxiety (DASS-anxiety > 15), respectively. Symptoms of depression and anxiety were more prevalent among females and less educated patients. Factors independently associated with both depressive and anxiety symptoms were post-traumatic stress disorder symptoms, low level of self-esteem, high somatic symptoms, low physical and mental health component scores, active smoking, physical inactivity, and longer disease duration. Patients with depressive and anxiety symptoms also reported poor social support and lower resilience.

Conclusion: There was a high level of depression and anxiety in this sample of cardiac patients. The results point to characteristics of patients in particular need for mental health screening and suggest possible targets for intervention such as strengthening of social support and of physical activity. The integration of mental health services into cardiac rehabilitation in Palestine and comparable cultural settings is warranted from the time of first diagnosis and onward.

Keywords: Depression, Anxiety, Cardiovascular diseases, Predictors, Prevalence, Cardiac rehabilitation

\footnotetext{
* Correspondence: nicole.probst@swisstph.ch

†'S. Haj-Yahia and N. Probst-Hensch contributed equally to this work.

${ }^{1}$ Department of Epidemiology and Public Health, Swiss Tropical and Public

Health Institute, Socinstrasse 57, P.O. Box, 4002 Basel, Switzerland

${ }^{2}$ University of Basel, Petersplatz 1, 4001 Basel, Switzerland

Full list of author information is available at the end of the article
}

(c) The Author(s). 2019 Open Access This article is distributed under the terms of the Creative Commons Attribution 4.0 International License (http://creativecommons.org/licenses/by/4.0/), which permits unrestricted use, distribution, and reproduction in any medium, provided you give appropriate credit to the original author(s) and the source, provide a link to the Creative Commons license, and indicate if changes were made. The Creative Commons Public Domain Dedication waiver (http://creativecommons.org/publicdomain/zero/1.0/) applies to the data made available in this article, unless otherwise stated. 


\section{Background}

Cardiovascular diseases (CVDs) and depression are among the leading causes of the global disease burden [1]. CVDs are the most common cause of death accounting for 17.9 million deaths globally, in 2015 [2]. Depression affects over 300 million people around the world [3], and is expected to become the main cause of disability globally, in 2030 [4]. Similarly, in 2010, anxiety affected approximately 272 million people, worldwide [5].

There is a high prevalence of mental disorders, particularly depression and anxiety, in CVD patients [6]. The bidirectional link between CVDs and these mental disorders has been extensively documented in literature [7-9]. Approximately, $15-30 \%$ of patients with CVD suffer from depressive disorders [10-14]. These rates of depression are two to three times higher than in the general population [15]. Moreover, depression and anxiety have been found to worsen prognosis and quality of life in patients with coronary artery disease (CAD), myocardial infarction (MI), heart failure (HF), unstable angina, and coronary artery bypass grafting (CABG) [16-22]. CABG is defined as a surgical procedure that is performed to treat people who have severe coronary heart disease, which improves blood flow to the heart [23]. They were also found to be the biggest driver of health care costs in coronary heart disease (CHD) patients [24].

Mental health problems have a direct physiological effect on the course of cardiac disease and their adverse effect may be mediated by non-compliance to lifestyle interventions, treatment and medication [25, 26]. Furthermore, these mental disorders add to the burden of managing CVD, from a perspective of treatment complexity and emotional distress. This problem is further aggravated by the high rate of additional co-morbidities such as diabetes, hypertension and obesity [27].

The American Heart Association (AHA) has recommended routine depression screening in cardiac patients [26]. However, health systems have not yet adequately responded and less than 15\% of cardiac patients are being diagnosed and treated for depression [28]. Integrating mental health into cardiac treatment is of particular relevance in low-middle-income countries (LMICs), where the burden of depression and anxiety is often high and aggravated by adverse life and political conditions. Similarly, mental health problems are stigmatized in many of these non-western cultures [29]. Little is known on the prevalence of depression and anxiety among cardiac patients in LMICs [30]. This also applies to Palestine, where mental disorders commonly are not recognized, diagnosed or treated, despite the increase in their prevalence [31]. Unaddressed mental care needs may be an important barrier to the successful management of cardiac patients in Palestine, where CVD remains the leading cause of death [32].
Mental health problems have not been studied in cardiac patients in the Palestinian population. Therefore, the aim of the current study was to determine the proportion of patients with depression and anxiety symptoms admitted with a cardiac diagnosis, to one of the four main hospitals in Nablus, Palestine. To guide physicians in effective mental health screening in the future, we also identified socio-demographic, clinical, psychosocial, and lifestyle factors associated with a high risk of depression and anxiety symptoms.

\section{Methods}

\section{Study design and population}

This cross-sectional hospital-based study was conducted on patients consecutively admitted to the cardiology and cardiac surgery departments of An-Najah National University Hospital, Arab-Specialized Hospital, Watani Hospital and Nablus Specialized Hospital in the Northern West Bank city of Nablus, Palestine. Patients were eligible for the study if they were between 30 and 80 years of age and had an existing or newly confirmed cardiac diagnosis warranting hospitalization during the period between March and November 2017. In the present study, cardiac diagnoses considered included CAD, ST elevation or non-ST elevation MI, angina, HF, cardiac arrhythmia, valve disease or any other cardiac disease. Diagnoses were confirmed using hospital medical charts. Patients were excluded if they had a normal cardiac catheterization (CATH), an acute or past stroke, end-stage kidney disease (including dialysis patients), peripheral vascular disease, major co-morbidities affecting mental health (alcohol abuse, drug abuse), neurological disorders (dementia, Alzheimer's disease, epilepsy, Parkinson's disease), cognitive impairment, a severe clinically diagnosed psychiatric condition or any other condition affecting the quality of their responses.

Eligible patients were recruited for in-person interviews by trained medical research assistants. Patients were identified using hospital registries and medical records. Interviews were conducted while patients were awaiting treatment (after CATH) or after their treatment, within 1 week of their admission to the hospital. Eligible patients were informed about the study objectives and benefits and written informed consent was obtained from those who agreed to participate. The study was approved by the Ethics Committee of Nordwest-und Zentral Schweiz (EKNZ) in Basel, Switzerland, and by the Institutional Review Board (IRB) committee at An-Najah National University in Nablus, Palestine.

\section{Study assessments and measures}

Data was collected using a structured questionnaire consisting of two parts (Tables 1 and 2; see Additional file 1 for more detailed description). The first part included 
detailed socio-demographic and clinical information obtained from patients' administrative and medical charts.

The second part was administered during a private interview and consisted of a sequence of screening instruments. These validated tools have demonstrated to be suitable for clinical populations to assess for depression and anxiety symptoms, QoL, post-traumatic stress disorder (PTSD), social support, resilience, self-esteem, somatic symptoms and lifestyle behaviors. The questionnaire, including the instruments, was translated from English to Arabic and back-translated from Arabic to English by two bilingual experts.

\section{Statistical analyses}

The primary endpoints in the present study were depression and anxiety. Descriptive statistics for stress as an endpoint are presented in an Additional file 2: Table S2, with no further description. The four predictor blocks investigated for association with depression and anxiety were socio-demographic, clinical, psychosocial and lifestyle factors (Table 1). Endpoints and predictors were described as means and standard deviations (SD) for quantitative variables and as absolute values and percentages for categorical variables. Differences in predictor variables according to presence or absence of depression and anxiety symptoms were described using chi-squared test and the Wilcoxon rank sum test, as appropriate. Fisher's exact test was used for results presenting a frequency below five. Multivariate ordered logistic regression analyses were performed to examine the independent association between predictor variables and depression or anxiety symptoms. All variables were entered in each of the models at once. Results are presented in separate models for depression and anxiety and are expressed as odds ratio (OR) and 95\% confidence intervals (95\% CI). The cut-offs for the respective outcome variables were normal, mild/moderate, and severe/very severe according to standardized cut-offs. Correlations between the outcome variables and other instruments used in the study were assessed using Spearman's rank correlation coefficient. Cronbach alpha was used to assess the internal consistency of the different scales. Statistical significance was defined as a two-sided $p$-value $<0.05$. All data was analyzed using the STATA Data Analysis and Statistical Software, version 14 .

\section{Results}

In total, 1092 patients were eligible and approached for an interview. Among the 1053 (96\%) patients which agreed to participate in the study, 1022 patients with complete outcome and predictor information were used in the analysis.

\section{Characteristics of study population}

Characteristics of the participants are presented in Table 3.

\section{Socio-demographic factors}

Among the 1022 patients, $73.4 \%$ were males. The mean age of patients was $58.9 \pm 10.1$ years (range $30-80$ years). The majority of participants were married (90.6\%), 37\% were unemployed, and $58.7 \%$ did not have a high school diploma. Most of the study population lived in cities $(46.4 \%)$ or villages $(46.3 \%)$, while $7.3 \%$ resided in refugee camps.

\section{Clinical factors}

The primary diagnoses among the sample were MI (39.7\%), CAD (32.7\%), angina (15.9\%) and other diagnoses (11.7\%), including mitral or aortic valve stenosis, valve regurgitation, heart block and others. Among the category of other diagnoses, $29(2.8 \%)$ patients had heart failure. Over $60 \%$ of participants had a previous cardiac diagnosis and had been diagnosed with a cardiac disease for 1 year or less. About half of participants underwent a CATH with a stent $(52.2 \%)$, while others underwent a CATH with a CABG (23.5\%), or some kind of other procedure (24.3\%), which was not yet performed at the time of the interview. Forty one percent of participants reported having two or more co-morbidities (mainly diabetes and hypertension) and $72.2 \%$ were on three or more medications. In addition, more than half of the participants' reported a family history of CVD. Approximately $37 \%$ of participating patients exhibited high somatic symptoms

Table 1 Predictor blocks used in bivariate and ordered logistic regression analyses

\begin{tabular}{ll}
\hline Block & Variable \\
\hline 1 Socio-demographic factors & Age, gender, marital status, residence, education level, occupation \\
2 Clinical factors & $\begin{array}{l}\text { Current diagnosis, previous cardiac diagnoses, years with cardiac disease, cardiac treatment (at admission), } \\
\text { Co-morbidities, medications, somatic symptoms (PHQ-15), family history of CVD, QoL (SF-12-PCS) }\end{array}$ \\
3 Psychosocial factors & PTSD (PTSD-PCL-S), social support (ESSI), resilience (RS-14), self-esteem, QOL (SF-12-MCS) \\
4 Lifestyle factors & Smoking status, currently on diet, fat consumption, vegetable and fruit consumption, alcohol use, physical activity, BMI
\end{tabular}

Note. CVD Cardiovascular disease, QoL Quality of life, PHQ-15 Patient health questionnaire-15, PCS Physical component summary, PTSD Post-traumatic stress disorder, PTSD-PCL-S Post-traumatic stress disorder checklist, ESSI ENRICHD Social support instrument, RS-14 Resilience scale-14, MCS Mental component summary, $B M I$ Body mass index 
Table 2 Study Instruments (see Additional file 1 for further descriptions)

\begin{tabular}{|c|c|}
\hline Instrument & Description \\
\hline \multicolumn{2}{|l|}{$\begin{array}{l}\text { Outcomes - assessment of } \\
\text { depression, anxiety and stress }\end{array}$} \\
\hline Cardiac Depression (CDS) & $\begin{array}{l}\text { The primary outcome of the study was measured using the Cardiac Depression Scale (CDS) by Hare et al., a } \\
\text { disease-specific, } 26 \text {-item questionnaire used to measure depression in patients with CVDs. CDS scores range from } \\
26 \text { to } 182 \text {, and items are scored on a seven-point Likert scale from } 1 \text { (strongly disagree) to } 7 \text { (strongly agree) [60]. } \\
\text { The CDS can be used as a continuous measure, where higher scores indicate higher depressive symptoms or as } \\
\text { an ordinal indicator of possible depression using cut-off points previously used in literature. In this study, the } \\
\text { presence of mild-to-moderate depression was defined as a CDS score of } 90-100 \text { and the presence of severe de- } \\
\text { pression as a score }>100 \text {. Scores below } 90 \text { indicated no-to-minimal depression [63]. }\end{array}$ \\
\hline $\begin{array}{l}\text { Depression, anxiety, stress } \\
\text { (DASS-42) }\end{array}$ & $\begin{array}{l}\text { Depression (DASS-depression), anxiety (DASS-anxiety) and stress (DASS-stress) were measured using the } \\
\text { Depression Anxiety Stress Scale-42 (DASS-42) by Lovibond \& Lovibond, a 42-item questionnaire consisting of } \\
\text { three subscales, each containing } 14 \text { items scored on a four-point Likert scale ranging from } 0 \text { (did not apply to } \\
\text { me at all) to } 3 \text { (applied to me very much), measuring the extent to which each item was experienced over the } \\
\text { past week. The scores are classified as: depression 0-9 (normal), 10-20 (mild-moderate), }>21 \text { (severe-very severe); } \\
\text { anxiety 0-7 (normal), 8-14 (mild-moderate), > }>15 \text { (severe-very severe); stress 0-14 (normal); 15-25 (mild-moder- } \\
\text { ate), > } 26 \text { (severe-very severe) [64]. }\end{array}$ \\
\hline \multicolumn{2}{|l|}{$\begin{array}{l}\text { Predictors - correlates of } \\
\text { depression and anxiety }\end{array}$} \\
\hline Somatic Symptoms (PHQ-15) & $\begin{array}{l}\text { The Patient Health Questionnaire (PHQ-15) is a 15-item somatic symptom scale derived from the full Patient- } \\
\text { Health-Questionnaire to measure the severity of somatization in patients [65]. }\end{array}$ \\
\hline $\begin{array}{l}\text { Quality of life (SF-12-PCS; SF-12- } \\
\text { MCS) }\end{array}$ & $\begin{array}{l}\text { Quality of life was assessed using the 12-item Short-Form Health Survey (SF-12), which is a generic measure of } \\
\text { overall health status. The SF-12 is comprised of two components, the Mental Component Summary (MCS) score } \\
\text { and the Physical Component Summary (PCS) score [66]. }\end{array}$ \\
\hline $\begin{array}{l}\text { Post-traumatic stress disorder } \\
\text { (PTSD-PCL-S) }\end{array}$ & $\begin{array}{l}\text { The Post-Traumatic Stress Disorder Checklist (PTSD-PCL-S) is a 17-item scale used to assess PTSD symptoms based } \\
\text { on the Diagnostic and Statistical Manual of Mental Disorders (DSM IV) criteria. The PTSD-PCL-S is used to link } \\
\text { symptom endorsements to a specific stressful or traumatic event or experience [67]. }\end{array}$ \\
\hline Social Support (ESSI) & $\begin{array}{l}\text { The ENRICHD (Enhancing Recovery in Coronary Heart) Social Support Instrument (ESSI) is a seven-item scale com- } \\
\text { prised from the Medical Outcomes Survey (MOS). It assesses four components of social support including emo- } \\
\text { tional, instrumental, informational and appraisal }[68,69] \text {. }\end{array}$ \\
\hline Self-esteem (SE) & $\begin{array}{l}\text { The Single-Item Self-Esteem Scale is a one-item scale developed as an alternative of the Rosenburg Self-Esteem } \\
\text { scale [70]. }\end{array}$ \\
\hline Resilience (RS-14) & Resilience Scale (RS-14) is a 14-item questionnaire that assesses individual resilience in a general population [71]. \\
\hline
\end{tabular}

on the PHQ-15. The mean score of the participants on the SF-12- PCS score was $37.6 \pm 12.4$.

\section{Psychosocial factors}

Forty Percent of patients reported having PTSD symptoms on the PTSD-PCL-S. Social support was generally high (64\%) among participants, according to the ESSI. Almost half of the participants presented with moderate to moderately-high resilience. The mean score for self-esteem was $5.8 \pm 1.4$, while the mean score for the SF-12-MCS was $39.7 \pm 13.2$.

\section{Lifestyle factors}

Almost half of the participants were current smokers while $35.6 \%$ had never smoked before. The vast majority of participants $(83.4 \%)$ reported not being on a diet, $47 \%$ low fat consumption, and $55.5 \%$ high vegetable and fruit consumption. The reported alcohol consumption was very low with $95 \%$ of participants not consuming any alcohol. Almost half of the patients reported no-to-minimal daily physical activity. Eighty percent of patients were either overweight or obese.

\section{Proportion of patients with depressive and anxiety symptoms at different severity levels}

Table 4 shows the proportion of patients with depression (CDS), depression (DASS-depression), anxiety (DASS-anxiety) and stress (DASS-stress) symptoms at different severity levels. Cutoffs for the levels of CDS and DASS subscales are also presented in Table 4. Based on our findings, the mean \pm SD depression score on the CDS was $101.3 \pm 15.6$ and the overall proportion of patients with depression was $78.7 \%$. According to the recommended cutoffs for the CDS, 21.3, 25.2 and 53.5\% of the sample had no, mild-to-moderate and severe-to-very severe depression symptoms, respectively. The means \pm SDs on the DASS-42 were 9.4 $\pm 8.6,9.4 \pm 6.8$ and $15.2 \pm 9$ for depression, anxiety, and stress, respectively. It was found that the overall proportion was 52.9, 53.1 and $37.4 \%$ for the presence of depressive, anxiety and stress symptoms according to the DASS-42. Based on recommended cutoffs, 47.1, 33.4 and $19.5 \%$ of patients reported normal, mild-to-moderate and severeto-very severe depressive symptoms (DASS-depression). In addition, $46.9 \%$ patients did not report any anxiety, 
Table 3 Socio-demographic, clinical, psychosocial and lifestyle characteristics of study population, $(n=1022)$

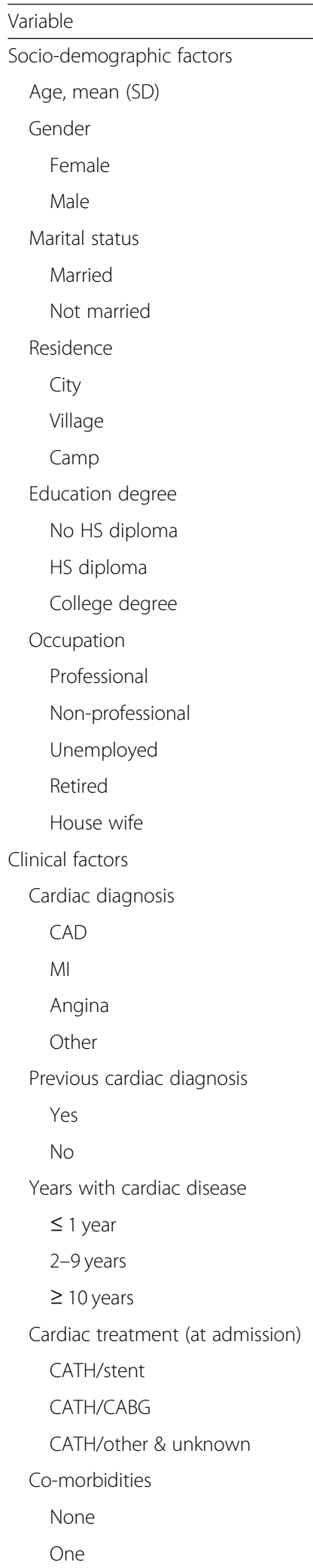

Two or more

$n(\%)$

$58.9 \pm 10.1$

272 (26.6)

750 (73.4)

926 (90.6)

96 (9.4)

474 (46.4)

473 (46.3)

$75(7.3)$

600 (58.7)

167 (16.3)

$255(25.0)$

209 (20.5)

$307(30.0)$

$378(37.0)$

81 (7.9)

47 (4.6)

334 (32.7)

406 (39.7)

162 (15.9)

$120(11.7)$

690 (67.5)

332 (32.5)

628 (61.5)

255 (24.9)

139 (13.6)

$534(52.2)$

$240(23.5)$

$248(24.3)$

299 (29.3)

303 (29.6)

$420(41.1)$
Table 3 Socio-demographic, clinical, psychosocial and lifestyle characteristics of study population, $(n=1022)$ (Continued)

\begin{tabular}{ll}
\hline Variable & $\mathrm{n}(\%)$ \\
\hline Medications & $132(12.9)$ \\
None & $152(14.9)$ \\
$1-2$ & $738(72.2)$ \\
$3-4$ & \\
Somatic symptoms (PHQ-15) & $91(8.9)$ \\
Minimal & $241(23.6)$ \\
Low & $314(30.7)$ \\
Medium & $376(36.8)$ \\
High &
\end{tabular}

Family history

Yes

$614(60.1)$

No

$408(39.9)$

QoL, (SF-12-PCS score),

mean (SD)

$37.6 \pm 12.4$

Psychosocial factors

PTSD (PTSD-PCL-S)

Minimal

$611(59.7)$

Some

$95(9.3)$

Moderate

$253(24.8)$

High

$63(6.2)$

Social support (ESSI)

Low

364 (35.6)

High

$658(64.4)$

Resilience (RS)

very low

$92(9.0)$

Low

103 (10.1)

Low-end

204 (20.0)

Moderate

257 (25.1)

Moderately-high

274 (26.8)

High

$92(9.0)$

Self-esteem (SE) score,

$5.8 \pm 1.4$

mean (SD)

QoL,(SF-12-MCS score),

$39.7 \pm 13.2$ mean (SD)

Lifestyle factors

Smoking status

Never

364 (35.6)

Former

170 (16.6)

Current

488 (47.8)

Currently on diet

Yes

$170(16.6)$

$\mathrm{No}$

$852(83.4)$

Fat consumption

Low 
Table 3 Socio-demographic, clinical, psychosocial and lifestyle characteristics of study population, $(n=1022)$ (Continued)

\begin{tabular}{ll}
\hline Variable & $\mathrm{n}(\%)$ \\
\hline Medium & $323(31.6)$ \\
High & $219(21.4)$ \\
$\begin{array}{l}\text { Vegetable \& fruit } \\
\text { consumption }\end{array}$ & \\
Low & $102(10.0)$ \\
Medium & $353(34.5)$ \\
High & $567(55.5)$ \\
Alcohol use, $(n=1019)$ & \\
Yes & $49(4.8)$ \\
No & $973(95.2)$ \\
Physical activity & \\
None & $337(33.0)$ \\
Not daily & $189(18.5)$ \\
Daily & $496(48.5)$ \\
\hline
\end{tabular}

Note. HS High school, MI Myocardial infarction, CAD Coronary artery disease, CATH Catheterization, CABG Coronary artery bypass graft, CVD Cardiovascular disease, PHQ-15 Patient health questionnaire-15, PCS Physical component summary, QoL Quality of life, SD Standard deviation, PTSD Post-traumatic stress disorder, PTSD-PCL-S Post-traumatic stress disorder checklist, ESSI ENRICHD social support instrument, RS-14 Resilience scale-14, MCS Mental component summary, BMI Body mass index

while $33.9 \%$ reported mild-to-moderate anxiety and $19.2 \%$ reported severe-to-very severe anxiety symptoms. According to the stress scale, $62.6 \%$ reported having no stress, $30 \%$ reported having mild-moderate stress symptoms and $7.4 \%$ severe-very severe stress symptoms. Patients that had mild-moderate and severe-very severe symptoms of depression or anxiety according to the DASS-42 were more likely to have severe depressive symptoms on the CDS. Nevertheless, 8 (4.0\%) and 9 (4.6\%) patients without any signs of depressive symptoms on the CDS, showed symptoms of depression and anxiety, respectively, on the DASS-42.

\section{Unadjusted correlations between continuous scores of the scales used in study}

Spearman correlation coefficients were calculated between the CDS and other instruments used in the study (see Fig. 1). The correlation of the CDS with the DASSdepression was $=0.57(\mathrm{p}=<0.001)$, with DASS-stress $=0.51(p<0.001)$, and with DASS-anxiety $=0.50(\mathrm{p}<$ 0.001). The correlations between the DASS subscales were as follows: DASS-depression and DASS-anxiety (0.65, p < 0.001); DASS-depression and DASS-stress (0.63, $\mathrm{p}<0.001)$; DASS-anxiety and DASS-stress $(0.61, \mathrm{p}$ $<0.001)$. CDS was positively correlated with the PHQ-15 $(0.44, p<.001)$ and the PTSD-PCL-S $(0.37, \mathrm{p}<.001)$ and the DASS-depression was also positively correlated with the PTSD-PCL-S $(0.57, \mathrm{p}<.001)$.

The above cluster of positively associated factors were weakly correlated to the physical and mental QoL components of the SF-12, social support, resilience, and self-esteem, which were strongly correlated among each other. Specifically, weak correlations were observed between CDS and the SF-12-MCS $(-0.39, \mathrm{p}<0.001)$, SF-12-PCS $(-0.29, \mathrm{p}<0.001)$, ESSI $(-0.12 \mathrm{p}<0.001)$, RS-14 $(-0.24, \mathrm{p}<0.001)$ and with the self-esteem scale $(-0.23, p<0.001)$.

Table 4 Proportion of patients with CDS-depression, DASS-depression, DASS-anxiety, DASS-stress at different severity levels $(n=$ 1022)

\begin{tabular}{|c|c|c|c|c|}
\hline & \multicolumn{4}{|c|}{ Depression, anxiety, stress according to CDS levels } \\
\hline & $\begin{array}{l}\text { Normal } \\
\mathrm{n}(\%)\end{array}$ & $\begin{array}{l}\text { Mild- Moderate } \\
\mathrm{n}(\%)\end{array}$ & $\begin{array}{l}\text { Severe-very severe } \\
\mathrm{n}(\%)\end{array}$ & $\begin{array}{l}\text { Percentage above } \\
\text { normal level }\end{array}$ \\
\hline CDS & $\begin{array}{l}\text { CDS }<90 \\
218(21.3)\end{array}$ & $\begin{array}{l}\text { CDS 90-100 } \\
257(25.2)\end{array}$ & $\begin{array}{l}\text { CDS > 100 } \\
547(53.5)\end{array}$ & 78.7 \\
\hline DASS-depression ( $\mathrm{D} \geq 10)$ & $481(47.1)$ & $341(33.4)$ & $200(19.5)$ & 52.9 \\
\hline Normal (0-9) & $166(34.5)$ & $164(35.1)$ & $151(31.4)$ & \\
\hline Mild/moderate (10-20) & $44(12.9)$ & $82(24.1)$ & $215(63.1)$ & \\
\hline Severe/very severe (21-42) & $8(4.0)$ & $11(5.5)$ & $181(90.5)$ & \\
\hline DASS-anxiety, $(A \geq 7)$ & $479(46.9)$ & $347(33.9)$ & $196(19.2)$ & 53.1 \\
\hline Normal (0-6) & $152(31.7)$ & $164(34.2)$ & $163(34.0)$ & \\
\hline Mild/moderate (7-14) & $57(16.4)$ & $77(22.2)$ & $213(61.4)$ & \\
\hline Severe/very severe (15-42) & $9(4.6)$ & $16(8.2)$ & $171(87.2)$ & \\
\hline DASS-stress, $(S \geq 15)$ & $640(62.6)$ & $307(30.0)$ & $75(7.4)$ & 37.4 \\
\hline Normal (0-14) & $178(27.8)$ & $206(32.2)$ & $256(40.0)$ & \\
\hline Mild/moderate (15-25) & $36(11.7)$ & $44(14.3)$ & $227(73.9)$ & \\
\hline Severe/very severe (26-42) & $4(5.3)$ & $7(9.3)$ & $64(85.3)$ & \\
\hline
\end{tabular}




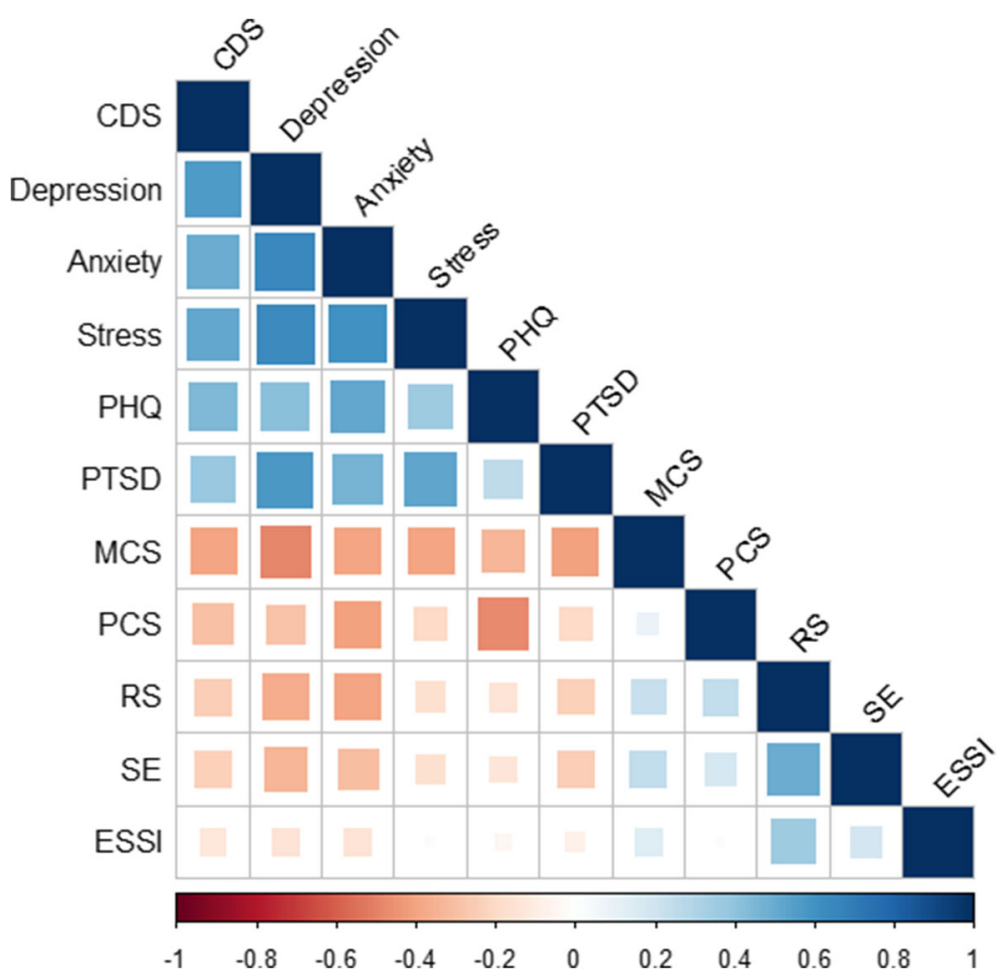

Fig. 1 Spearmen correlations between CDS, DASS-depression, DASS-anxiety, DASS-stress and other instruments used in the study. Note. CDS = Cardiac Depression Scale; PHQ = Patient Health Questionnaire-15; PTSD = Post-Traumatic Stress Disorder; MCS = Mental Component Summary; PCS = Physical Component Summary; RS = Resilience Scale-14; SE = Self-esteem; ESSI = ENRICHD Social Support Instrument. Blue colors represent positive correlations and red colors represent negative correlations. Correlations measured are expressed as rho spearman.

\section{Association between socio-demographic, clinical, psychosocial, lifestyle factors and depressive and anxiety symptoms}

The bivariate distribution of socio-demographic, clinical, psychological, and lifestyle characteristics according to the presence or absence of depression symptoms (CDS), anxiety symptoms (DASS-anxiety) and stress symptoms (DASS-stress) is presented in Additional file 3: Table S1 (depression (CDS) and anxiety) and Additional file 2: Table S2 (stress). Briefly, with regard to socio-demographic factors, bivariate analysis revealed depressive and anxiety symptoms were more frequent among females than males (depression: $84.9 \%$ vs. $76.4 \%, p=0.003$; anxiety: $72.4 \%$ vs. $55.6 \%, \mathrm{p}=<0.001)$ and among those with lower educational level (depression: $81.0 \%$ [no high school diploma] vs. $78.0 \%$ [high school diploma] and $71.1 \%$ [college degree], $p=0.02$; anxiety: $63.2 \%$ [no high school diploma] vs. $59.8 \%$ (high school diploma) and $52.8 \%$ (college degree), $p<0.05)$. Both, depressive and anxiety symptoms were most prevalent among those unemployed and housewives (depression: $\mathrm{p}<0.001$; anxiety: $\mathrm{p}<0.001$ ).

Multivariable ordered logistic regression was performed to determine the independent association of factors in the four predictor blocks with a) depressive symptoms, categorized as no depressive symptoms, moderate depressive symptoms, and severe depressive symptoms according to the CDS and b) anxiety symptoms, categorized as minimal anxiety symptoms, mild-moderate anxiety symptoms, and severe-severe anxiety symptoms according to the DASS-anxiety subscale. The results for the four blocks of variables are presented in Table 5.

Overall, most of the psychosocial factors were consistently associated with both, depression and anxiety. Participants with depression or anxiety were more likely to exhibit at least some symptoms of PTSD. Odds ratios tended to be higher for anxiety than for depression (moderate symptoms vs. minimal symptoms: $\mathrm{OR}_{\text {depresssion }} 1.87$ (95\% CI 1.29-2.71) vs. OR anxiety 3.01 (95\% CI 2.12-4.27). Patients with depression or anxiety had a lower score for the mental component of QoL (SF-12-MCS) $\left[\mathrm{OR}_{\text {depression }}\right.$ 0.96 (95\% CI 0.95-0.97); OR anxiety 0.98 (95\% CI $0.97-$ 0.99)]. High resilience and high social support were inversely associated with depression and anxiety [high vs. low social support: $\mathrm{OR}_{\text {depression }} 0.71$ (95\% CI 0.52-0.97); $\mathrm{OR}_{\text {anxiety }} 0.74(95 \% \mathrm{CI} 0.54-1.00)$ ]. The inverse association with resilience tended to be stronger in the presence of anxiety [high vs. very low resilience: $\mathrm{OR}_{\text {depression }} 0.42$ (95\% CI 0.18-0.94); OR anxiety 0.22 (95\% CI 0.11-0.48)].

In addition to psychosocial factors, the physical component of QoL (SF-12-PCS) and somatic symptoms 
Table 5 Factors associated with DEPRESSION and ANXIETY in multivariate ordered logistic regression

\begin{tabular}{|c|c|c|c|c|c|c|}
\hline \multirow[t]{2}{*}{ Variable } & \multicolumn{3}{|c|}{ Depression (CDS) } & \multicolumn{3}{|c|}{ Anxiety (DASS-anxiety) } \\
\hline & $\overline{\mathrm{OR}}$ & $95 \% \mathrm{Cl}$ & $P$ value & $\overline{\mathrm{OR}}$ & $95 \% \mathrm{Cl}$ & $P$ value \\
\hline \multicolumn{7}{|l|}{ Socio-demographic factors } \\
\hline Age, mean (SD) & 0.99 & $(0.98-1.00)$ & 0.393 & 0.96 & $(0.95-0.99)$ & $<0.001$ \\
\hline \multicolumn{7}{|l|}{ Gender } \\
\hline Female & (reference) & & & (reference) & & \\
\hline Male & 0.83 & $(0.51-1.36)$ & 0.469 & 0.68 & $(0.43-1.10)$ & 0.117 \\
\hline \multicolumn{7}{|l|}{ Marital status } \\
\hline Married & (reference) & & & (reference) & & \\
\hline Not married & 0.38 & $(0.22-0.65)$ & 0.001 & 1.28 & $(0.76-2.13)$ & 0.350 \\
\hline \multicolumn{7}{|l|}{ Residence } \\
\hline City & (reference) & & & (reference) & & \\
\hline Village & 1.22 & $(0.91-1.64)$ & 0.191 & 0.74 & $(0.54-1.00)$ & 0.054 \\
\hline Camp & 1.35 & $(0.76-2.40)$ & 0.308 & 0.56 & $(0.31-1.01)$ & 0.054 \\
\hline \multicolumn{7}{|l|}{ Education degree } \\
\hline No HS diploma & (reference) & & & (reference) & & \\
\hline HS diploma & 1.15 & $(0.82-1.62)$ & 0.421 & 0.93 & $(0.65-1.31)$ & 0.666 \\
\hline College degree & 1.19 & $(0.76-1.87)$ & 0.449 & 1.09 & $(0.68-1.76)$ & 0.715 \\
\hline \multicolumn{7}{|l|}{ Occupation } \\
\hline Professional & (reference) & & & (reference) & & \\
\hline Non-professional & 0.85 & $(0.55-1.31)$ & 0.472 & 1.15 & $(0.73-1.81)$ & 0.544 \\
\hline Unemployed & 1.12 & $(0.67-1.88)$ & 0.662 & 0.89 & $(0.54-1.48)$ & 0.660 \\
\hline Retired & 0.71 & $(0.38-1.33)$ & 0.289 & 1.44 & $(0.75-2.73)$ & 0.269 \\
\hline House wife & 0.33 & $(0.14-0.80)$ & 0.014 & 0.65 & $(0.27-1.56)$ & 0.335 \\
\hline \multicolumn{7}{|l|}{ Clinical Factors } \\
\hline \multicolumn{7}{|l|}{ Cardiac diagnosis } \\
\hline$C A D$ & 0.89 & $(0.63-1.25)$ & 0.508 & 0.75 & $(0.53-1.07)$ & 0.111 \\
\hline Ml & (reference) & & & (reference) & & \\
\hline Angina & 1.26 & $(0.83-1.92)$ & 0.271 & 0.54 & $(0.35-0.86)$ & 0.009 \\
\hline Other & 0.82 & $(0.49-1.37)$ & 0.440 & 1.23 & $(0.76-1.99)$ & 0.407 \\
\hline \multicolumn{7}{|l|}{ Previous cardiac diagnosis } \\
\hline Yes & 0.67 & $(0.47-0.96)$ & 0.028 & 1.52 & $(1.05-2.21)$ & 0.026 \\
\hline No & (reference) & & & (reference) & & \\
\hline \multicolumn{7}{|l|}{ Years with cardiac disease } \\
\hline$\leq 1$ year & (reference) & & & (reference) & & \\
\hline 2-9 years & 1.46 & $(1.00-2.13)$ & 0.048 & 1.10 & $(0.76-1.59)$ & 0.663 \\
\hline$\geq 10$ years & 1.71 & $(1.06-2.75)$ & 0.028 & 0.95 & $(0.60-1.50)$ & 0.832 \\
\hline \multicolumn{7}{|c|}{ Cardiac treatment (at admission) } \\
\hline CATH/stent & (reference) & & & (reference) & & \\
\hline $\mathrm{CATH} / \mathrm{CABG}$ & 1.42 & $(0.98-2.04)$ & 0.065 & 0.89 & $(0.62-1.29)$ & 0.558 \\
\hline CATH/other \& unknown & 1.34 & $(0.95-1.89)$ & 0.097 & 1.44 & $(1.02-2.04)$ & 0.036 \\
\hline \multicolumn{7}{|l|}{ Co-morbidities } \\
\hline None & (reference) & & & (reference) & & \\
\hline 1 & 0.93 & $(0.64-1.34)$ & 0.695 & 0.96 & $(0.65-1.43)$ & 0.860 \\
\hline $2+$ & 1.39 & $(0.94-2.04)$ & 0.096 & 1.36 & $(0.92-2.01)$ & 0.127 \\
\hline
\end{tabular}


Table 5 Factors associated with DEPRESSION and ANXIETY in multivariate ordered logistic regression (Continued)

\begin{tabular}{|c|c|c|c|c|c|c|}
\hline \multirow[t]{2}{*}{ Variable } & \multicolumn{3}{|c|}{ Depression (CDS) } & \multicolumn{3}{|c|}{ Anxiety (DASS-anxiety) } \\
\hline & $\overline{\mathrm{OR}}$ & $95 \% \mathrm{Cl}$ & $P$ value & $\overline{\mathrm{OR}}$ & $95 \% \mathrm{Cl}$ & $P$ value \\
\hline \multicolumn{7}{|l|}{ Medications } \\
\hline None & (reference) & & & (reference) & & \\
\hline $1-2$ & 1.33 & $(0.78-2.29)$ & 0.293 & 1.20 & $(0.68-2.10)$ & 0.521 \\
\hline $3-4$ & 1.19 & $(0.74-1.92)$ & 0.470 & 1.31 & $(0.79-2.18)$ & 0.299 \\
\hline \multicolumn{7}{|c|}{ Somatic symptoms (PHQ-15) } \\
\hline Minimal & (reference) & & & (reference) & & \\
\hline Low & 1.18 & $(0.72-1.94)$ & 0.506 & 1.41 & $(0.74-2.72)$ & 0.296 \\
\hline Medium & 1.86 & $(1.12-3.08)$ & 0.015 & 3.35 & $(1.76-6.35)$ & $<0.001$ \\
\hline High & 3.00 & $(1.73-5.18)$ & $<0.001$ & 7.64 & $(3.96-14.78)$ & $<0.001$ \\
\hline \multicolumn{7}{|l|}{ Family history } \\
\hline Yes & 1.03 & $(0.77-1.38)$ & 0.824 & 0.76 & $(0.57-1.03)$ & 0.075 \\
\hline No & (reference) & & & (reference) & & \\
\hline QoL, (SF-12-PCS score) & 0.98 & $(0.97-1.00)$ & 0.015 & 0.97 & $(0.96-0.99)$ & $<0.001$ \\
\hline \multicolumn{7}{|l|}{ Psychosocial factors } \\
\hline \multicolumn{7}{|l|}{ PTSD (PTSD-PCL-S) } \\
\hline Minimal & (reference) & & & (reference) & & \\
\hline Some & 1.32 & $(0.83-2.10)$ & 0.247 & 2.34 & $(1.47-3.71)$ & $<0.001$ \\
\hline Moderate & 1.87 & $(1.29-2.71)$ & 0.001 & 3.01 & $(2.12-4.27)$ & $<0.001$ \\
\hline High & 1.28 & $(0.00)$ & 0.974 & 7.37 & $(3.89-14.0)$ & $<0.001$ \\
\hline \multicolumn{7}{|l|}{ Social support (ESSI) } \\
\hline Low & (reference) & & & (reference) & & \\
\hline High & 0.71 & $(0.52-0.97)$ & 0.032 & 0.74 & $(0.54-1.00)$ & 0.049 \\
\hline \multicolumn{7}{|l|}{ Resilience (RS-14) } \\
\hline Very low & (reference) & & & (reference) & & \\
\hline Low & 1.05 & $(0.49-2.25)$ & 0.896 & 0.76 & $(0.41-1.41)$ & 0.381 \\
\hline Low-end & 0.78 & $(0.39-1.56)$ & 0.479 & 0.43 & $(0.25-0.77)$ & 0.004 \\
\hline Moderate & 0.60 & $(0.30-1.21)$ & 0.154 & 0.37 & $(0.20-0.66)$ & 0.001 \\
\hline Moderately-high & 0.48 & $(0.23-0.98)$ & 0.044 & 0.19 & $(0.10-0.36)$ & $<0.001$ \\
\hline High & 0.42 & $(0.18-0.94)$ & 0.035 & 0.22 & $(0.11-0.48)$ & $<0.001$ \\
\hline Self-esteem (SE) score & 0.97 & $(0.85-1.10)$ & 0.640 & 0.85 & $(0.76-0.96)$ & 0.007 \\
\hline QoL,(SF-12-MCS score) & 0.96 & $(0.95-0.97)$ & $<0.001$ & 0.98 & $(0.97-0.99)$ & $<0.001$ \\
\hline \multicolumn{7}{|l|}{ Lifestyle factors } \\
\hline \multicolumn{7}{|l|}{ Smoking status } \\
\hline Never & (reference) & & & (reference) & & \\
\hline Former & 1.87 & $(1.17-2.97)$ & 0.008 & 0.71 & $(0.44-1.14)$ & 0.154 \\
\hline Current & 1.61 & $(1.11-2.33)$ & 0.013 & 1.37 & $(0.93-2.02)$ & 0.106 \\
\hline \multicolumn{7}{|l|}{ Currently on diet } \\
\hline Yes & 0.59 & $(0.40-0.86)$ & 0.007 & 1.45 & $(0.98-2.13)$ & 0.060 \\
\hline No & (reference) & & & (reference) & & \\
\hline \multicolumn{7}{|l|}{ Fat consumption } \\
\hline Low & (reference) & & & (reference) & & \\
\hline Medium & 0.73 & $(0.53-1.00)$ & 0.054 & 1.09 & $(0.79-1.51)$ & 0.583 \\
\hline High & 0.79 & $(0.55-1.14)$ & 0.206 & 1.28 & $(0.88-1.86)$ & 0.187 \\
\hline
\end{tabular}


Table 5 Factors associated with DEPRESSION and ANXIETY in multivariate ordered logistic regression (Continued)

\begin{tabular}{|c|c|c|c|c|c|c|}
\hline \multirow[t]{2}{*}{ Variable } & \multicolumn{3}{|c|}{ Depression (CDS) } & \multicolumn{3}{|c|}{ Anxiety (DASS-anxiety) } \\
\hline & $\mathrm{OR}$ & $95 \% \mathrm{Cl}$ & $P$ value & $\mathrm{OR}$ & $95 \% \mathrm{Cl}$ & $P$ value \\
\hline \multicolumn{7}{|c|}{ Vegetable \& fruit consumption } \\
\hline Low & (reference) & & & (reference) & & \\
\hline Medium & 0.83 & $(0.50-1.37)$ & 0.466 & 0.52 & $(0.31-0.86)$ & 0.011 \\
\hline High & 0.73 & $(0.45-1.19)$ & 0.207 & 0.80 & $(0.49-1.30)$ & 0.375 \\
\hline \multicolumn{7}{|l|}{ Alcohol use } \\
\hline Yes & 1.31 & $(0.66-2.61)$ & 0.439 & 1.19 & $(0.63-2.24)$ & 0.591 \\
\hline No & (reference) & & & (reference) & & \\
\hline \multicolumn{7}{|l|}{ Physical activity } \\
\hline None & (reference) & & & (reference) & & \\
\hline Not daily & 0.64 & $(0.42-0.98)$ & 0.040 & 0.57 & $(0.37-0.87)$ & 0.009 \\
\hline Daily & 0.43 & $(0.30-0.60)$ & $<0.001$ & 0.98 & $(0.70-1.38)$ & 0.908 \\
\hline \multicolumn{7}{|l|}{ BMl } \\
\hline Underweight & 0.63 & $(0.05-7.7)$ & 0.720 & 2.09 & $(0.22-19.9)$ & 0.522 \\
\hline Normal weight & (reference) & & & (reference) & & \\
\hline Overweight & 0.81 & $(0.55-1.18)$ & 0.272 & 0.61 & $(0.42-0.89)$ & 0.010 \\
\hline Obese & 0.95 & $(0.63-1.43)$ & 0.824 & 0.87 & $(0.59-1.29)$ & 0.495 \\
\hline
\end{tabular}

Note. Multivariate ordered logistic regression reported for socio-demographic, clinical, psychosocial and lifestyle factors associated with depression, CDS (not depressed; mild-moderate depression; severely-very severe depression) and DASS-anxiety (no anxiety, mild-moderate anxiety; severe-very severe anxiety). Analyses were performed in two separate models for depression and anxiety, mutually adjusting for all factors in the four predictor blocks in both models. Analyses are also adjusted for the hospital to which patients were admitted. OR Odds ratio, $\mathrm{Cl}$ Confidence Interval, SD Standard deviation, HS High school, MI Myocardial infarction, CAD Coronary artery disease, CATH Catheterization, CABG Coronary artery bypass graft, PHQ-15 Patient health questionnaire-15, PCS Physical component summary, PTSD Post-traumatic stress disorder, PTSD-PCL-S Post-traumatic stress disorder checklist, ESSI ENRICHD social support instrument, RS-14 Resilience scale-

14, MCS Mental component summary, BMI Body mass index; $P$ values in bold are significant at $p<0.05$

(PHQ-15) also showed consistent associations with both depression and anxiety [high vs. minimal somatic symptoms: OR depression 3.00 (95\% CI 1.73-5.18); OR anxiety 7.64 (95\% CI 3.96-14.78)]; [SF-12-PCS: OR depression 0.98 (95\% CI 0.97-1.00); OR anxiety $0.97 \quad$ (95\% CI $0.96-0.99)]$. Current smoking was associated with depression with the strongest odds ratio observed for depression and former smoking: OR 1.87 (95\% CI 1.17-2.97). Finally, patients with depression and anxiety were more likely to be physically inactive compared to patients without the respective psychological problems.

A few factors exhibited associations with only one of the two mental health outcomes. For example, patients residing in villages or camps were less likely to show symptoms of anxiety compared to patients living in the

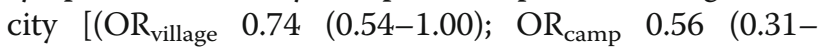
1.01)]. Unemployment was positively associated with depression, but not anxiety. The presence of previous cardiac diagnoses was positively associated with anxiety, but inversely associated with depression [ $\left(\mathrm{OR}_{\mathrm{depression}}\right.$ 0.67 (95\% 0.47-0.96); OR $\mathrm{OR}_{\text {anxiety }} 1.52$ (95\% $1.05-2.21$ )]. Symptoms of depression were more frequent among patients with a cardiac diagnosis for more than 10 years compared to patients with a diagnosis for a year or less [years since first diagnosis $\geq 10$ vs. $\leq 1$ year OR 1.71 (95\% 1.06-2.75)]. Patients who were diagnosed with angina were more likely to have anxiety symptoms than those diagnosed with an MI. Underweight and obese participants were more likely to exhibit symptoms of anxiety than those of normal weight and overweight.

\section{Reliability assessment (Cronbach's alpha) of the study instruments}

Cronbach $\alpha$ for the primary outcome variable, CDS was 0.86 and 0.92 (DASS-depression), 0.82 (DASS-anxiety), and 0.89 (DASS-stress) for the DASS subscales, respectively, indicating high consistency for the relevant psychometric scales. This indicates all scales exhibit acceptable internal consistency with little likelihood of item redundancy. Inter-item correlations between the 26 items of the CDS and the total CDS scores ranged from 0.08 to 0.58 , and all correlations were statistically significant at the 0.01 level. Cronbach $\alpha$ for the other scales used in the study was: 0.78 for PHQ-15, 0.86 for PTSD-PCL-S, 0.82 for ESSI and 0.88 for RS- 14 .

\section{Discussion}

In the present study, the observed rates of depressive and anxiety symptoms were high. Only $21 \%$ (CDS) and 46\% (DASS-anxiety) of patients did not exhibit any symptoms of depression and anxiety, respectively. Our findings point to the need for integrating mental health care into cardiac 
treatment. It is noteworthy that several factors found to be associated with depression and anxiety may serve as screening and possibly as intervention targets.

Rates of mental health problems reported in earlier studies for patients with different cardiac diagnoses and in different cultural and health system settings ranged from 14 to $73 \%$ [33-38] for depressive symptoms and 15 to $48 \%$ [33-37] for anxiety symptoms. These varying rates are explained in part by differences in sample sizes, the instruments and cutoffs used for classifying depression and anxiety and the type of cardiac disease targeted in studies.

Lower rates of depression than in the current study were observed in other settings including Norway (14\%) [33], USA (15\%) [39], Brazil (26.4\%) [34] and Pakistan (14\%) [36]. Similar among these studies, was the common psychiatric instruments used to assess for depression, all of which were not specific for cardiac populations. In contrast, a different study assessing depression using the CDS found a rate of $73.2 \%$ of severe depression in Iranian patients with acute coronary syndrome (ACS), a rate even higher than in this study [38].

Similar anxiety rates to the current study were observed in Iran (28.5\%) [37] and Pakistan (18\%) [36]. Interestingly, in another study conducted in Brazil, Meneghetti et al. found a very high prevalence of $48.4 \%$ for anxiety symptoms among ACS patients using the Hospital Anxiety and Depression Scale (HADS) [34]. A study in the USA also reported, $37 \%$ of patients with MI due to spontaneous coronary artery dissection screened for anxiety using the Generalized Anxiety Disorder 7-Item Scale (GAD-7) [35].

Mental health problems are generally high in the Palestinian population [31]. In the absence of a healthy control group the results of this study do not allow to conclude that depression and anxiety are more common in cardiac patients. However, cardiac patients are in particular need of treatment for depression and anxiety given that existing evidence points to their adverse effect on the course of heart disease. Furthermore, cardiac rehabilitation may be an efficient starting point to address mental health issues beyond the patient and to the extended family and social network. Given the shortage of mental health services available and the local economic instability in Palestine, the provision of additional services needs to be implemented in a cost-effective way. The identification of subgroups of cardiac patients at higher risk of depression and anxiety can guide screening and interventions.

In perspective of mental health screening among cardiac patients, focus should be given to females and less educated patients. The higher rates of depression and anxiety seen in these sub-groups were previously described in literature [34, 37, 40, 41]. Women seem to be more vulnerable to the trauma caused by cardiac events, which leads to a deterioration in depression and anxiety symptoms [37]. As observed in some [34, 42, 43], but not all studies [37, 38, 44], the association between gender and social status may not be direct as suggested by the disappearance of gender and social status differences in the fully adjusted models [34, 37, 38, 42].

The presence of the following additional characteristics in cardiac patients should be a red flag for cardiologists to consider mental health care in cardiac practice: symptoms of PTSD, low levels of self-esteem, somatic symptoms, low QoL components, active smoking, physical inactivity, and longer disease duration. In contrast, a high level of resilience seems to reduce symptoms of psychological problems, as previously observed in patients with heart failure $[45,46]$. Unlike findings reported previously, comorbidities were not consistently more common in the presence of mental health disorders [47]. Little is known about the association of PSTD symptoms with depression and anxiety in cardiac patients. A study conducted on 813 patients who received angiograms at a large U.S. Veterans Administration Medical Center found depression to be positively associated with PTSD, smoking and alcohol consumption [48]. Low MCS and PCS scores on the SF-12, smoking, and chest pain were recently identified as the strongest predictors of longitudinally sustained high levels of depression and anxiety in CHD patients [24].

Factors serving as targets for intervention include smoking, physical activity and social support. Smoking cessation interventions are crucial for cardiac rehabilitation, however in the presence of depression, results are less successful and interventions may need to be adapted [49]. Sedentary behavior, a risk factor for depression in the general population [50], was previously associated with depression according to the Beck Depression Inventory-II in patients hospitalized for ACS [51]. In a small non-randomized intervention study with heart failure patients, aerobic interval training decreased symptoms of depression over a period of 12-weeks [52]. In studies on breast cancer [53], promotion of physical activity may have the additional benefit of improving selfesteem, a factor associated with depression and anxiety in this study and a predictor of mortality in the general population [54]. In addition, according to previous studies $[43,55-58]$, the inverse association between high social support and low levels of anxiety and depression points to another important target for prevention as it is supported by firm evidence from previous studies. Poor social support among patients with ACS was observed in secondary analyses of a randomized trial to reduce the effectiveness of treatment with antidepressants [59]. The quality of social support plays an important role, as overprotective behaviors of partners can have an adverse effect [58]. Interestingly, in the current study marital status and social support were associated with presence of 
depressive symptoms independently and in opposite directions.

The current study has several strengths. First, it utilized a broad set of validated instruments to identify depression and anxiety symptoms as well as associated factors. The overall validity of the CDS in this study was almost similar to levels originally reported by Hare and Davis [60]. The validity of the DASS-42 was satisfactory, in line with other findings of other studies, including those originally reported by Lovibond and Lovibond [61]. Furthermore, the CDS is the only psychometric scale suitable for the comparative depression assessment in heart disease patients, subjected to different interventions $[60,62]$. This is evident in the present study, as depression rates were lower when assessed by the DASS-42. The CDS, also has excellent properties for the diagnosis of MDD, a score of $\geq 95$ having a $97 \%$ sensitivity at $85 \%$ specificity (AUC 0.96) [62]. Second, the large sample size provided sufficient statistical power for testing independent associations. Third, the study subjects are well characterized, which allowed for addressing confounding. Fourth, the high participation rate decreased the likelihood of selection and participation bias. Finally, the findings of the study despite being hospitalbased are likely generalizable to the entire cardiac patient population in the city of Nablus and surrounding cities of Palestine, considering the study sites provide cardiac care to a large percentage of cardiac patients in the area.

Nonetheless, the cross-sectional nature of the study does not enable us to make causal inferences. A long-term follow-up is foreseen to investigate the predictive effect of the study characteristics and predictors with regard to the course of depression, anxiety and heart disease. While the reliability of the previously validated instruments was confirmed in our study, the instruments have not been validated specifically for the context of cardiac patients in Palestine. Furthermore, some of the risk factors could only be captured broadly to avoid lengthy interviews. This likely caused some misclassification, as in the case of physical activity. Recall bias may have added to the misclassification of risks, given the retrospective nature of the interview. Also, the study was not sufficiently powered to investigate differences in the frequency of depression and anxiety as well as associated factors between relevant subgroups of clinical diagnoses, for example MI and HF. Finally, some patients were recruited at the time of admission and for the most part before receiving their intervention or diagnosis, and thus were under much pressure and stress. The level of depression and anxiety could have been overestimated and may decrease in part over the course of disease. The most appropriate time point to assess depression and anxiety from a prognostic perspective is unknown.

\section{Conclusions}

The alarmingly high rate of depression and anxiety symptoms observed in cardiac patients in Palestine points to the need for integrating mental health care into cardiac rehabilitation. The prognostic value of depression and anxiety with regard to the course of heart disease, adherence to treatment and quality of life needs to be investigated. Treatment of psychological problems from the disease onset and onwards is crucial considering longer disease duration puts individuals at higher risk of being depressed. The expertise of social scientists and medical anthropologists is needed for identifying efficient means to overcome barriers related to the stigmatization of psychological disorders.

\section{Additional files}

Additional file 1: Description of study instruments. (DOCX 39 kb)

Additional file 2: Table S2. Socio-demographic, clinical, psychosocial, lifestyle factors by STRESS status, $(n=1022)$. (DOCX 24 kb)

Additional file 3: Table S1. Socio-demographic, clinical, psychosocial, lifestyle factors by DEPRESSION and ANXIETY status, $(n=1022)$. (DOCX $24 \mathrm{~kb})$

\section{Abbreviations}

ACS: Acute coronary syndrome; AHA: American Heart Association; BMI: Body mass index; $C A B G$ : Coronary artery bypass grafting; CAD: Coronary artery disease; CATH: Catheterization; CDS: Cardiac Depression Scale; CHD: Coronary heart disease; Cl: Confidence interval; CVD: Cardiovascular disease; CVDs: Cardiovascular diseases; DASS-42: Depression Anxiety Stress Scale-42; DSM-IV: Diagnostic and Statistical Manual of Mental Disorders-IV; EKNZ: Ethics Committee of Nordwest-und Zentral Schweiz; ENRICHD: Enhancing Recovery in Coronary Heart; ESSI: ENRICHD Social Support Instrument; HF: Heart failure; HS: High school; IRB: Institutional Review Board; LMIC: Low-middle income countries; MCS: Mental Component Summary; MDD: Major depressive disorder; MI: Myocardial infarction; MOS: Medical Outcomes Survey; OR: Odds ratio; PCS: Physical Component Summary; PHQ-15: Patient Health

Questionnaire-15; PTSD: Post-traumatic stress disorder; PTSD-PCL-S: PostTraumatic Stress Disorder Checklist; QOL: Quality of life; RS-14: Resilience Scale-14; SD: Standard deviation; SE: Self-esteem; SF-12: The 12-item Short Form Health Survey

\section{Acknowledgements}

Professor Marcel Tanner (Swiss Tropical and Public Health Institute, Basel, Switzerland) initiated the collaboration between An-Najah National University and the Swiss Tropical and Public Health Institute. Professor Sabina de Geest (Director Institute of Nursing Science, University of Basel, Switzerland) provided highly valuable professional expertise and input for the manuscript.

\section{Funding}

The study was funded by a Swiss Government Excellence Fellowship for Foreign Scholars (ESKAS) for the PhD student, HA, working on the project and by the Freiwillige Akademische Gesellschaft, Basel, Switzerland. The funders of the project took no part in the design of the study, data collection and analysis, interpretation of the data or the writing of the manuscript.

\section{Availability of data and materials}

The datasets used and/or analyzed during the current study are available from the corresponding author on reasonable request.

\section{Authors' contributions}

HA conducted data collection, analyzed and interpreted data, and wrote the manuscript. AAlka supervised the data collection and the implementation of 
the study in Palestine and edited the manuscript. AAlkh, AH, HO, JS, MT assisted in data collection. EZ and SHY contributed to designing the study. EZ and CS participated in planning the data analysis. CS supervised the statistical analysis. EZ, SHY, and CS contributed to the interpretation of the results. NPH designed the study, directed its implementation, data analysis and result interpretation. She edited all versions of the manuscript. All authors read and approved the final manuscript

\section{Ethics approval and consent to participate}

This study was approved by the Ethics Committee of Nordwest- und Zentral Schweiz (EKNZ) in Basel, Switzerland and by the Institutional Review Board (IRB) committee at An-Najah National University in Nablus, Palestine. All patients enrolled in the study provided written informed consent

\section{Consent for publication}

Not applicable.

\section{Competing interests}

The authors declare that they have no competing interests.

\section{Publisher's Note}

Springer Nature remains neutral with regard to jurisdictional claims in published maps and institutional affiliations.

\section{Author details}

'Department of Epidemiology and Public Health, Swiss Tropical and Public Health Institute, Socinstrasse 57, P.O. Box, 4002 Basel, Switzerland. ${ }^{2}$ University of Basel, Petersplatz 1, 4001 Basel, Switzerland. ${ }^{3}$ Faculty of Medicine and Health Sciences, An-Najah National University, Rafidia Street, P.O. Box 7, Nablus, Palestine. ${ }^{4}$ An-Najah National University Hospital, Asira Street, Nablus, Palestine. ${ }^{5}$ School of Clinical Sciences, University of Bristol, 69 St Michael's Hill, Bristol BS2 8DZ, UK. ${ }^{6}$ Institute of Cardiovascular and Medical Sciences, Glasgow University, 126 University Place, Glasgow G12 8TA, UK.

Received: 28 October 2018 Accepted: 18 February 2019

Published online: 26 February 2019

\section{References}

1. Murray CJ, Vos T, Lozano R, Naghavi M, Flaxman AD, Michaud C, Ezzati M, Shibuya K, Salomon JA, Abdalla S, et al. Disability-adjusted life years (DALYS) for 291 diseases and injuries in 21 regions, 1990-2010: a systematic analysis for the global burden of disease study 2010. Lancet. 2012;380:2197-223.

2. Roth GA, Johnson C, Abajobir A, Abd-Allah F, Abera SF, Abyu G, Ahmed M, Aksut B, Alam T, Alam K, et al. Global, regional, and National Burden of cardiovascular diseases for 10 causes, 1990 to 2015. J Am Coll Cardiol. 2017:70:1-25

3. WHO. Depression fact sheet. 2012. http://www.who.int/news-room/factsheets/detail/depression. Accessed 25 Sept 2018.

4. Mensah GA, Collins PY. Understanding mental health for the prevention and control of cardiovascular diseases. Glob Heart. 2015;10:221-4.

5. Baxter AJ, Vos T, Scott KM, Norman RE, Flaxman AD, Blore J, Whiteford HA. The regional distribution of anxiety disorders: implications for the global burden of disease study, 2010. Int J Methods Psychiatr Res. 2014;23:422-38.

6. Rutledge T, Linke SE, Krantz DS, Johnson BD, Bittner V, Eastwood JA, Eteiba W, Pepine CJ, Vaccarino V, Francis J, et al. Comorbid depression and anxiety symptoms as predictors of cardiovascular events: results from the NHLBIsponsored Women's ischemia syndrome evaluation (WISE) study. Psychosom Med. 2009;71:958-64.

7. Lippi G, Montagnana M, Favaloro EJ, Franchini M. Mental depression and cardiovascular disease: a multifaceted, bidirectional association. Semin Thromb Hemost. 2009;35:325-36.

8. Holt RIG, Phillips DIW, Jameson KA, Cooper C, Dennison EM, Peveler RC, Hertfordshire Cohort Study G. The relationship between depression, anxiety and cardiovascular disease: findings from the Hertfordshire cohort study. J Affect Disord. 2013;150:84-90.

9. Riba MWL, Rubenfire M. Psychiatry and heart disease: the mind, brain, and heart. Hoboken: Wiley-Blackwell; 2011.

10. Lesperance F, Frasure-Smith N. Depression in patients with cardiac disease: a practical review. J Psychosom Res. 2000;48:379-91.

11. Frasure-Smith N, Lesperance F. Depression and coronary artery disease. Herz. 2006;31(Suppl 3):64-8.
12. Rudisch B, Nemeroff CB. Epidemiology of comorbid coronary artery disease and depression. Biol Psychiatry. 2003;54:227-40.

13. Thombs BD, de Jonge P, Coyne JC, Whooley MA, Frasure-Smith N, Mitchell AJ, Zuidersma M, Eze-Nliam C, Lima BB, Smith CG, et al. Depression screening and patient outcomes in cardiovascular care: a systematic review. JAMA. 2008;300:2161-71.

14. Carney RM, Freedland KE. Depression in patients with coronary heart disease. Am J Med. 2008;121:S20-7.

15. Kessler RC, Berglund P, Demler O, Jin R, Koretz D, Merikangas KR, Rush AJ, Walters EE, Wang PS. The epidemiology of major depressive disorder: results from the National Comorbidity Survey Replication (NCS-R). JAMA. 2003;289: 3095-105.

16. Lett HS, Blumenthal JA, Babyak MA, Sherwood A, Strauman T, Robins C, Newman MF. Depression as a risk factor for coronary artery disease: evidence, mechanisms, and treatment. Psychosom Med. 2004;66:305-15.

17. Nicholson A, Kuper $H$, Hemingway $H$. Depression as an aetiologic and prognostic factor in coronary heart disease: a meta-analysis of 6362 events among 146538 participants in 54 observational studies. Eur Heart J. 2006; 27:2763-74.

18. Rutledge T, Reis VA, Linke SE, Greenberg BH, Mills PJ. Depression in heart failure a meta-analytic review of prevalence, intervention effects, and associations with clinical outcomes. J Am Coll Cardiol. 2006;48:1527-37.

19. Jiang W, Alexander J, Christopher E, Kuchibhatla M, Gaulden LH, Cuffe MS, Blazing MA, Davenport C, Califf RM, Krishnan RR, O'Connor CM. Relationship of depression to increased risk of mortality and rehospitalization in patients with congestive heart failure. Arch Intern Med. 2001;161:1849-56.

20. de Jonge P, Spijkerman TA, van den Brink RH, Ormel J. Depression after myocardial infarction is a risk factor for declining health related quality of life and increased disability and cardiac complaints at 12 months. Heart. 2006:92:32-9

21. Blumenthal JA, Lett HS, Babyak MA, White W, Smith PK, Mark DB, Jones R, Mathew JP, Newman MF. Depression as a risk factor for mortality after coronary artery bypass surgery. Lancet. 2003;362:604-9.

22. de Jager TAJ, Dulfer K, Radhoe S, Bergmann MJ, Daemen J, van Domburg RT, Lenzen MJ, Utens E. Predictive value of depression and anxiety for longterm mortality: differences in outcome between acute coronary syndrome and stable angina pectoris. Int J Cardiol. 2018;250:43-8.

23. Harris $\mathrm{R}$, Croce B, Tian DH. Coronary artery bypass grafting. Ann Cardiothorac Surg. 2013;2:579.

24. Palacios J, Khondoker M, Mann A, Tylee A, Hotopf M. Depression and anxiety symptom trajectories in coronary heart disease: associations with measures of disability and impact on 3-year health care costs. J Psychosom Res. 2018;104:1-8.

25. Chaddha A, Robinson EA, Kline-Rogers E, Alexandris-Souphis T, Rubenfire M Mental health and cardiovascular disease. Am J Med. 2016:129:1145-8.

26. Lichtman JH, Bigger JT Jr, Blumenthal JA, Frasure-Smith N, Kaufmann PG, Lesperance F, Mark DB, Sheps DS, Taylor CB, Froelicher ES. Depression and coronary heart disease: recommendations for screening, referral, and treatment: a science advisory from the American Heart Association Prevention Committee of the Council on Cardiovascular Nursing, Council on Clinical Cardiology, Council on Epidemiology and Prevention, and Interdisciplinary Council on Quality of Care and Outcomes Research: endorsed by the American Psychiatric Association. Circulation. 2008:118: 1768-75.

27. Leon BM, Maddox TM. Diabetes and cardiovascular disease: epidemiology biological mechanisms, treatment recommendations and future research. World J Diabetes. 2015;6:1246-58.

28. Huffman JC, Smith FA, Blais MA, Beiser ME, Januzzi JL, Fricchione GL. Recognition and treatment of depression and anxiety in patients with acute myocardial infarction. Am J Cardiol. 2006;98:319-24.

29. Rathod S, Pinninti N, Irfan M, Gorczynski P, Rathod P, Gega L, Naeem F. Mental health service provision in low- and middle-income countries. Health Serv Insights. 2017;10:1178632917694350

30. Ormel J, Von Korff M, Burger H, Scott K, Demyttenaere K, Huang YQ, Posada-Villa J, Pierre Lepine J, Angermeyer MC, Levinson D, et al. Menta disorders among persons with heart disease - results from world mental health surveys. Gen Hosp Psychiatry. 2007;29:325-34

31. Marie M, Hannigan $B$, Jones A. Mental health needs and services in the West Bank, Palestine. Int J Ment Health Syst. 2016;10(1):23.

32. Mosleh M, Dalal K, Aljeesh $Y$. Burden of chronic diseases in the Palestinian health-care sector using disability-adjusted life-years. Lancet. 2018;391:S21. 
33. Hanssen TA, Nordrehaug JE, Eide GE, Bjelland I, Rokne B. Anxiety and depression after acute myocardial infarction: an 18-month follow-up study with repeated measures and comparison with a reference population. Eur J Cardiovasc Prev Rehabil. 2009;16:651-9.

34. Meneghetti CC, Guidolin BL, Zimmermann PR, Sfoggia A. Screening for symptoms of anxiety and depression in patients admitted to a university hospital with acute coronary syndrome. Trends Psychiatry Psychother. 2017; 39:12-8.

35. Liang JJ, Tweet MS, Hayes SE, Gulati R, Hayes SN. Prevalence and predictors of depression and anxiety among survivors of myocardial infarction due to spontaneous coronary artery dissection. J Cardiopulm Rehabil Prev. 2014;34: 138-42.

36. Akhtar MS, Malik SB, Ahmed MM. Symptoms of depression and anxiety in post-myocardial infarction patients. J Coll Physicians Surg Pak. 2004;14:615-8.

37. Bayani B, Yousefi S, Bayani M, Shirmohammadi M, Alimoradi A, Falsoleiman H, Yazdi N, Arbabi M. Depression and anxiety in a cardiovascular outpatient clinic: a descriptive study. Iran J Psychiatry. 2011;6:125-7.

38. Goudarzian AH, Nia HS, Tavakoli H, Soleimani MA, Yaghoobzadeh A, Tabari F, Taebei M. Prevalence of cardiac depression and its related factors among patients with acute coronary syndrome. Asian J Pharm Res Health Care. 2016:8(S1):30-5.

39. Moraska AR, Chamberlain AM, Shah ND, Vickers KS, Rummans TA, Dunlay SM, Spertus JA, Weston SA, McNallan SM, Redfield MM, Roger VL. Depression, healthcare utilization, and death in heart failure: a community study. Circ Heart Fail. 2013;6:387-94.

40. Dogar IA, Khawaja IS, Azeem MW, Awan H, Ayub A, lqbal J, Thuras P. Prevalence and risk factors for depression and anxiety in hospitalized cardiac patients in Pakistan. Psychiatry (Edgmont). 2008;5:38-41.

41. Alexandri A, Georgiadi E, Mattheou P, Polikandrioti M. Factors associated with anxiety and depression in hospitalized patients with first episode of acute myocardial infarction. Arch Med Sci Atheroscler Dis. 2017;2:e90-9.

42. Shokrgozar S, Allahi M, Ahmadi R, Khairkhah J, Moorosi M. Severity of depression in hospitalized patients with cardiovascular diseases by cardiac depression scale (CDS). J Guilan UnivMed Sci. 2015;24:9-15.

43. Su SF, Chang MY, He CP. Social support, unstable angina, and stroke as predictors of depression in patients with coronary heart disease. J Cardiovasc Nurs. 2018;33:179-86.

44. Al-Abbudi S, Faris L, Abd Wady Z. Prevalence and assessment of severity of depression among ischemic heart disease patients attending outpatient cardiology department Baghdad teaching Hospital, Baghdad, Iraq. J Psychiatry. 2018;21. https://doi.org/10.4172/2378-5756.1000438.

45. Toukhsati SR, Jovanovic A, Dehghani S, Tran T, Tran A, Hare DL. Low psychological resilience is associated with depression in patients with cardiovascular disease. Eur J Cardiovasc Nurs. 2017;16:64-9.

46. Chang LY, Wu SY, Chiang CE, Tsai PS. Depression and self-care maintenance in patients with heart failure: a moderated mediation model of self-care confidence and resilience. Eur J Cardiovasc Nurs. 2017:16:435-43.

47. Eng HS, Yean LC, Das S, Letchmi S, Yee KS, Bakar RA, Hung J, Choy CY. Anxiety and depression in patients with coronary heart disease: a study in a tertiary hospital. Iran J Med Sci. 2011;36:201-6.

48. Shankman SA, Nadelson J, McGowan SK, Sovari AA, Vidovich MI. The predictive power of depression screening procedures for veterans with coronary artery disease. Vasc Health Risk Manag. 2012;8:233-8.

49. Doyle F, Rohde D, Rutkowska A, Morgan K, Cousins G, McGee H. Systematic review and meta-analysis of the impact of depression on subsequent smoking cessation in patients with coronary heart disease: 1990 to 2013. Psychosom Med. 2014;76:44-57.

50. Hamer M, Stamatakis E. Prospective study of sedentary behavior, risk of depression, and cognitive impairment. Med Sci Sports Exerc. 2014:46:718-23.

51. Zhu Y, Blumenthal JA, Shi C, Jiang R, Patel A, Zhang A, Yu X, Gao R, Wu Y. Sedentary behavior and the risk of depression in patients with acute coronary syndromes. Am J Cardiol. 2018;121:1456-60.

52. Isaksen K, Munk PS, Giske R, Larsen Al. Effects of aerobic interval training on measures of anxiety, depression and quality of life in patients with ischaemic heart failure and an implantable cardioverter defibrillator: a prospective non-randomized trial. J Rehabil Med. 2016:48:300-6.

53. Segar ML, Katch VL, Roth RS, Garcia AW, Portner TI, Glickman SG, Haslanger S, Wilkins EG. The effect of aerobic exercise on self-esteem and depressive and anxiety symptoms among breast cancer survivors. Oncol Nurs Forum. 1998:25:107-13.
54. Stamatakis KA, Lynch J, Everson SA, Raghunathan T, Salonen JT, Kaplan GA. Self-esteem and mortality: prospective evidence from a population-based study. Ann Epidemiol. 2004;14:58-65.

55. Compare A, Zarbo C, Manzoni GM, Castelnuovo G, Baldassari E, Bonardi A, Callus E, Romagnoni C. Social support, depression, and heart disease: a ten year literature review. Front Psychol. 2013;4:384.

56. Bucholz EM, Strait KM, Dreyer RP, Geda M, Spatz ES, Bueno H, Lichtman JH, D'Onofrio G, Spertus JA, Krumholz HM. Effect of low perceived social support on health outcomes in young patients with acute myocardial infarction: results from the VIRGO (variation in recovery: role of gender on outcomes of young AMl patients) study. J Am Heart Assoc. 2014;3:e001252.

57. Notara V, Panagiotakos DB, Papataxiarchis E, Verdi M, Michalopoulou M, Tsompanaki E, Kogias Y, Stravopodis P, Papanagnou G, Zombolos S, et al. Depression and marital status determine the 10-year (2004-2014) prognosis in patients with acute coronary syndrome: the GREECS study. Psychol Health. 2015;30:1116-27.

58. Zarbo C, Compare A, Baldassari E, Bonardi A, Romagnoni C. In sickness and in health: a literature review about function of social. Clin Pract Epidemiol Ment Health. 2013;9:255-62.

59. Kim JW, Kang HJ, Bae KY, Kim SW, Shin IS, Yoon JS, Hong YJ, Ahn Y, Jeong $\mathrm{MH}$, Kim JM. Social support deficit and depression treatment outcomes in patients with acute coronary syndrome: findings from the EsDEPACS study. Int J Psychiatry Med. 2018:91217418791439. https://doi.org/10.1177/ 0091217418791439

60. Hare DL, Davis CR. Cardiac depression scale: validation of a new depression scale for cardiac patients. J Psychosom Res. 1996:40:379-86.

61. Lovibond PF, Lovibond SH. The structure of negative emotional states: comparison of the depression anxiety stress scales (DASS) with the Beck depression and anxiety inventories. Behav Res Ther. 1995;33:335-43.

62. Shi WY, Stewart AG, Hare DL. Major depression in cardiac patients is accurately assessed using the cardiac depression scale. Psychother Psychosom. 2010;79:391-2.

63. Wise FM, Harris DW, Carter LM. Validation of the cardiac depression scale in a cardiac rehabilitation population. J Psychosom Res. 2006;60:177-83.

64. Lovibond SHLP. Manual for the depression anxiety stress scales. 2nd ed. Sydney: Psychology Foundation; 1995

65. Kroenke K, Spitzer RL, Williams JB. The PHQ-15: validity of a new measure for evaluating the severity of somatic symptoms. Psychosom Med. 2002;64: 258-66.

66. Ware J Jr, Kosinski M, Keller SD. A 12-item short-form health survey: construction of scales and preliminary tests of reliability and validity. Med Care. 1996:34:220-33.

67. Blanchard EB, Jones-Alexander J, Buckley TC, Forneris CA. Psychometric properties of the PTSD checklist (PCL). Behav Res Ther. 1996:34:669-73.

68. The ENRICHD Investigators. Enhancing recovery in coronary heart disease patients (ENRICHD): study design and methods. The ENRICHD investigators. Am Heart J. 2000;139:1-9.

69. The ENRICHD Investigators. Enhancing recovery in coronary heart disease (ENRICHD) study intervention: rationale and design. Psychosom Med. 2001; 63:747-55.

70. Robins RW, Hendin HM, Trzesniewski KH. Measuring global self-esteem: construct validation of a single-item measure and the Rosenberg selfesteem scale. Personal Soc Psychol Bull. 2001;27:151-61.

71. Wagnild GM, Guinn PE. The resilience scale user's guide: for the U.S. english version of the resilience scale TM and the 14-item resilience scale TM (RS-14 TM). USA: Resilience Center; 2009

\section{Ready to submit your research? Choose BMC and benefit from:}

- fast, convenient online submission

- thorough peer review by experienced researchers in your field

- rapid publication on acceptance

- support for research data, including large and complex data types

- gold Open Access which fosters wider collaboration and increased citations

- maximum visibility for your research: over $100 \mathrm{M}$ website views per year

At $\mathrm{BMC}$, research is always in progress.

Learn more biomedcentral.com/submissions 